\title{
Health Care Economics: A Study Guide for Neuroradiology Fellows, Part 1
}

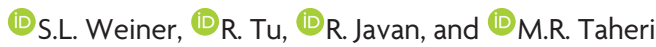

\begin{abstract}
SUMMARY: Few resources are available in the medical literature for a comprehensive review of current health care economics as it relates to radiologists, specifically framed by topics defined by the Accreditation Council for Graduate Medical Education in the evaluation of neuroradiology fellows. Therefore, we present a comprehensive review article as a study guide for fellows to learn from and gain competence in the Accreditation Council for Graduate Medical Education neuroradiology milestones on health care economics.
\end{abstract}

ABBREVIATIONS: ACGME = Accreditation Council for Graduate Medical Education; ASNR = American Society of Neuroradiology; CMS = Centers for Medicare and Medicaid Services; CPT = Current Procedural Terminology; ICD = International Statistical Classification of Diseases and Related Health Problems; MACRA = Medicare Access and Children's Health Insurance Program Reauthorization Act; MPPR = Multiple Procedure Payment Reduction; PC = professional component; RUC = American Medical Association Specialty Society Relative Value Scale Update Committee; RVS = Resource-Based Relative Value Scale; RVU = relative value unit; TDABC $=$ Time-Driven Activity-Based Costing; TC = technical component

$\mathbf{U}^{\prime}$ nderstanding and pragmatically applying the economic principles found in existing resources may be challenging for fellows, given the relatively fragmented dissemination of the principles in the literature. Therefore, we present a comprehensive review article as a study guide for fellows to learn from and gain competence in the ACGME neuroradiology milestones on health care economics. In addition, it is the authors' hope that this work might serve as a foundation for diagnostic radiology residents, other imaging subspecialty fellows, and practicing radiologists, facilitating implementation in real-world radiology practice. This review article primarily relates to Medicare and its unique role in the physician reimbursement process. The role of private payers is beyond the scope of this effort and will not be explored to any meaningful degree. Finally, this work will be presented as a 2-part review article, with Part 1 covering ACGME milestones 1-3 and Part 2 covering milestones 4-5.

\section{MATERIALS AND METHODS}

A search using the PubMed Medical Subject Heading terms "diagnostic imaging/economics" and "radiology/economics" per-

From the Neuroradiology Section, Department of Radiology, George Washington University Hospital, Washington, DC.

Paper previously presented, in part, as a digital exhibit at: Annual Meeting of the American Society of Neuroradiology, April 22-27, 2017; Long Beach, California.

Please address correspondence to M. Reza Taheri, MD, PhD, 900 23rd St, NW, G2092, George Washington University, Washington, DC 20037; e-mail: rtaheri@mfa.gwu.edu

- Indicates open access to non-subscribers at www.ajnr.org

http://dx.doi.org/10.3174/ajnr.A5381 formed in late 2016 resulted in a nonexhaustive compilation of approximately 50 review articles on current health care economics, nearly all of which were published within the past 5-6 years, many within the past $1-2$ years. From these, approximately 20 reference articles were used to synthesize a relatively comprehensive compendium of useful reference information on the topic, with the ACGME neuroradiology milestones on health care economics and systems-based practice serving as a framework.

\section{ACGME Neuroradiology Milestones on Health Care Economics and Systems-Based Practice: Defining the Levels}

The ACGME neuroradiology milestones on health care economics and systems-based practice are made up of 5 levels per the most recent iteration of The Neuroradiology Milestone Project from July 2015. ${ }^{1}$ In general, this project is part of a larger effort by the ACGME to assess the competence of residents and fellows in their respective specialties or subspecialties as they matriculate through their training program toward unsupervised practice. It provides a framework for assessing the development of trainees in several key elements of specialty/subspecialty competency across 6 core domains: medical knowledge, patient care, professionalism, interpersonal communication, practice-based learning, and system-based practice. More specifically, as a neuroradiology fellow progresses through his or her training program, the milestones serve as a benchmark for measuring competence in key elements related to the subspecialty of neuroradiology, one of which is health care economics. The 5 levels of each key element are presented as a developmental framework, moving from less advanced 


\begin{tabular}{lcclc}
\hline \multicolumn{1}{c}{ Level 1 } & Level 2 & Level 3 & Level 4 & Level $\mathbf{5}$ \\
\hline $\begin{array}{l}\text { Describes the technical } \\
\text { and professional }\end{array}$ & $\begin{array}{c}\text { Participates in departmental } \\
\text { cost-savings initiatives }\end{array}$ & $\begin{array}{c}\text { Describes billing and coding of } \\
\text { subspecialty-specific exams }\end{array}$ & $\begin{array}{c}\text { Describes Medicare } \\
\text { reimbursement }\end{array}$ & $\begin{array}{c}\text { Describes the roles } \\
\text { of the ACR and } \\
\text { components of }\end{array}$ \\
imaging costs in the & & and recognizes and corrects & for radiology & AMA in the \\
subspecialty division & & incorrect coding; creates & studies and & valuation and \\
& & reports that contain the & available bonus & revaluation of \\
& & elements necessary to & payments for & CPT codes \\
& & support exam coding & physicians & \\
\hline
\end{tabular}

Note:-ACR indicates American College of Radiology; AMA, American Medical Association.

a Reprinted from The Neuroradiology Milestone Project, The Accreditation Council for Graduate Medical Education and the American Board of Radiology.

(level 1) to more advanced (level 5). As the fellow progresses from entry into the training program to graduation, the goal is to move from novice to expert in the subspecialty, with level 4 (or above) being the target for graduation.

The 5 levels for measuring a neuroradiology fellow's competence in health care economics are outlined in Table 1 and are presented in order as major section headings (levels) in this article.

\section{Historical Background}

Under the Social Security Act of 1965 (P.L. 89-97, Approved July 30, 1965 [79 Stat. 286]; please refer to https://www.ssa.gov/OP_ Home/comp2/F089-097.html), physicians were reimbursed for both their professional work and any practice expenses directly related to that work on an as-billed fee-for-service basis. Modest oversight was provided to facilitate billing that was "usual, customary, and reasonable," though there were no established national guidelines to ensure uniformity, which resulted in a wide range of reimbursements. This indiscriminate scheme persisted until 1982 when the Tax Equity and Fiscal Responsibility Act was passed into law. It established the diagnosis-related group, which was a payment system in which hospitals were paid an established (fixed) prospective fee for the cost of inpatient care based on specific patient diagnoses. ${ }^{2,3}$ In 1983, Congress amended the Social Security Act to include a national diagnosis-related group-based hospital payment system for all patients on Medicare. Although Medicare costs stabilized via shorter hospital stays, radiologists' reimbursements leveled off or diminished; this change accelerated a trend at that time in which increasing numbers of radiologists were detaching themselves from hospital employment (and therefore the diagnosis-related group payment model). These radiologists began to set up professional corporations that contracted with hospitals and maintained separate billing arrangements. Although already prevalent, this program accelerated the separation of billing components; the professional fees are charged by the radiologists for the interpretation of inpatient imaging studies, and the technical fees are charged by the hospitals for the performance of the studies. ${ }^{2}$

However, in a setting of rising health care costs in the 1980s and burgeoning capitation of physician reimbursement, there was a call by Congress for greater scrutiny of the way government revenue was being distributed for medical services, which resulted in the creation of the Resource-Based Relative Value Scale (RVS), a constantly evolving entity that remains in use today. ${ }^{2}$ Congress commissioned William Hsaio, $\mathrm{PhD}$, of the Harvard School of Public Health to establish the RVS; he was, at the time, a national spokesperson on the concept. It estimated the discrete amount of work involved in providing specific medical services and ranked those services on the basis of the time needed to complete the work, the intensity of the work, and practice expense. Intensity was defined by technical/physical skill, mental effort/judgment, and stress, the latter being a gauge for the possibility of untoward legal action. The radiology community played an important role in the early adoption of the RVS system, having an RVS of its own as far back as 1963 at the request of the Department of Defense; this RVS became incorporated into the RVS used by all specialties. Broad adoption of the RVS was largely due to this early effort of the radiology community, which predated Dr Hsaio's effort, in concert with other specialties, resulting in its use by the greater medical community. ${ }^{2}$ A timeline is presented in the Figure, which highlights additional historical details.

\section{Level 1: Technical and Professional Components}

Defining the Terms. Radiology services comprise 2 distinct billable elements, the technical component (TC) and the professional component (PC). ${ }^{4}$ The TC is that portion of the global fee that reflects the cost of operating and maintaining the medical equipment, the cost of medical supplies, the cost of renting or purchasing the real estate that houses the equipment, the cost associated with having radiologic technologists who perform the examinations, and so forth. The PC refers to the cost associated with the radiologist's interpretation of the examination, which includes the written radiology report, which is the sum of the physician work, practice expense, and professional liability insurance inherent in the production of the report. In short, the technical fee reflects the cost of performing the study and the professional fee reflects the cost of interpreting the study, which vary on the basis of geographic location (Table 2).

Relative Value Unit. Many physicians, including radiologists, use relative value units (RVUs) as defined by Medicare as a measure of value in the Centers for Medicare and Medicaid Services (CMS) reimbursement formula for radiologist services. RVUs are typically the method used to allocate radiologist reimbursement, being based on the complexity, skill, and time required to perform a clinical treatment plan or procedure, which are elements related to the PC. In general, the TC amounts to a higher percentage of the global cost of a radiologic examination, due to the considerably larger overhead needed to maintain its various operational elements. For example, the current National Physician Fee Schedule Relative Value File breaks down a "brain MR imaging without contrast" in the Washington, DC area in the following manner: It 


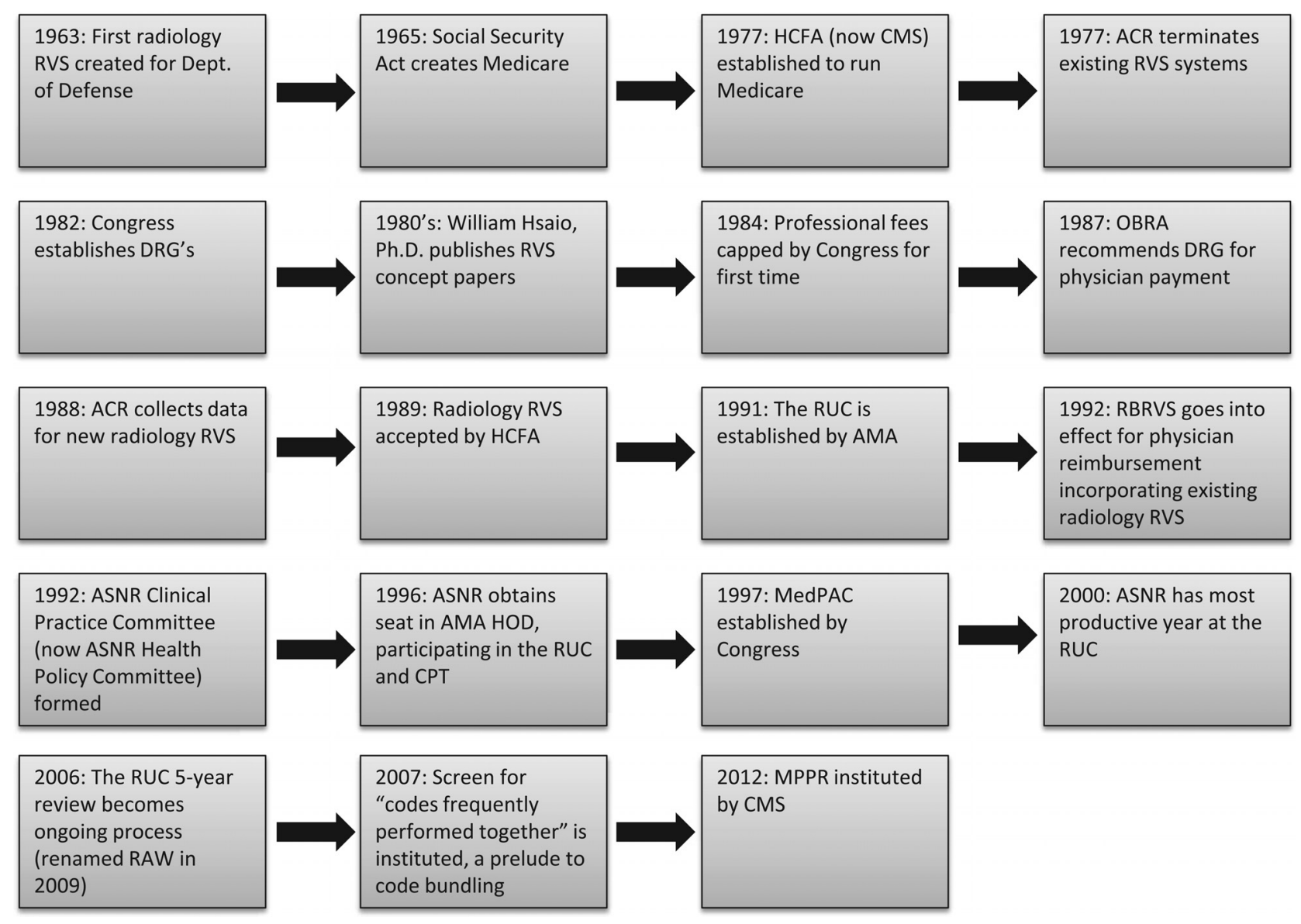

FIGURE. Timeline of the RVS. ACR indicates American College of Radiology; AMA, American Medical Association; HCFA, Health Care Financing Administration; HOD, House of Delegates; MedPAC, Medicare Payment Advisory Committee; OBRA, Omnibus Budget Reconciliation Act; RAW, Relativity Assessment Workgroup; DRG, diagnosis-related group; RBRVS, Resource-Based Relative Value Scale. Adapted with permission from Donovan. ${ }^{2}$

Table 2: Centers for Medicare and Medicaid Services 2017 CMS Component Fees for MRI Brain without Dye (HCPCS Code 70551) $)^{5,6}$

\begin{tabular}{lccc}
\hline & TC & PC & Global Fee \\
\hline Arkansas & $\$ 137.58$ & $\$ 71.88$ & $\$ 209.46$ \\
District of Columbia & $\$ 190.74$ & $\$ 83.10$ & $\$ 273.84$ \\
Minnesota & $\$ 160.56$ & $\$ 74.15$ & $\$ 234.71$ \\
\hline
\end{tabular}

Note:- - HCPCS indicates Healthcare Common Procedure Coding System.

is assigned 6.52 RVUs for the global payment, 4.41 RVUs for the technical component, and 2.11 RVUs for the professional component (1.48 RVUs for physician work). ${ }^{6}$

Basics of Current Procedural Terminology. Accurate and consistent physician reimbursement mandates a standardized language for medical procedures. In recognition of this need, the American Medical Association developed the Current Procedural Terminology (CPT; https://www.ama-assn.org/practice-management/ cpt-current-procedural-terminology) nomenclature in 1965 as a description of medical services and procedures. ${ }^{7}$ Initially, this manual focused on surgery, so radiology, much less neuroradiology, had little representation in the form of billable coding. In 1970 when the second edition was published, each code was expanded to 5 digits with the 70000-79999 code series applying to radiology. By the mid-to-late 1970s, the third and fourth editions were released, which contained increasingly detailed codes in keeping with the ever-growing complexity of the health care system. This procedural coding system has continued to evolve across the years, merging with other coding systems and via the Health Insurance Portability and Accountability Act of 1996, was updated to support standards for electronic transactions. These changes culminated in the establishment of the CPT-5 project, which resulted in nomenclature that facilitated new tracking procedures and specific reporting measures that could be used in performance-based payment. This expansion paved the way for the Centers for Medicare and Medicaid Services opting to formally incorporate CPT codes in Medicare claims processing. Finally, in 2000, CPT became the national coding standard for reporting medical services and procedures. ${ }^{7}$

The codes found in the CPT codebook are divided into 3 categories (Table 3). Category I codes are common to everyday clinical practice and are referred to the American Medical Association Specialty Society Relative Value Scale Update Committee for valuation. ${ }^{8}$ They require US Food and Drug Administration approval for any related drugs or devices, must have demonstrated clinical efficacy validated by peer-reviewed literature, and must be the standard of care practiced by multiple physicians in the United States. Category II codes are used to report quality performance initiatives and are designed to facilitate data collection, 
tracking of performance measures, and compliance with state and federal law, with minimal medical record review. They are not used for coding services or procedures that are billed. As Physician Quality Reporting System measures grow, the number of category II codes continues to increase (Physician Quality Reporting System will be more fully discussed later in this article). Category III codes were initially created in 2001 to track new or experimental procedures and technologies that aid the FDA in the approval process. They are temporary by definition, being approved for a 5 -year period with the option of extending this period once, and are not assigned a work value. Therefore, payment for category III codes is discretionary. Considering the scientific evidence, a category III code can be converted to a category I code before the expiration of the initial or renewal term. If the procedure proves ineffective at the end of the term, the code can die. Category I and III codes have different rigors; having a code does not ensure coverage and payment.

Two key committees are responsible for proposing new CPT codes and changes to the code set: the CPT Editorial Panel and the CPT Advisory Committee. The CPT Editorial Panel oversees the development of new and revised codes and governs the maintenance of code sets. ${ }^{7}$ The panel comprises physicians and other relevant stakeholders, including CMS representatives. The CPT Advisory Committee is made up of societal representatives from the American Medical Association House of Delegates and is the apparatus through which the American Society of Neuroradiology (ASNR) and other medical societies are represented. The Advisory Committee assists the CPT Editorial Panel by proposing code set changes and providing insight into coding proposals submitted by other interested stakeholders, whether they are other medical societies, industry vendors, insurance carriers, and so forth. These panels will be discussed later in this article.

\section{Controversies}

Multiple Procedure Payment Reduction. Multiple procedure payment reduction (MPPR) is a very controversial element of radiology reimbursements. ${ }^{4}$ This cost-saving scheme was first proposed by CMS in 2006 across multiple code families, including those related to radiologic imaging; it applies to advanced imaging

Table 3: Three categories of codes found in the CPT codebook

\begin{tabular}{ll}
\hline CPT Category & \multicolumn{1}{c}{ Description } \\
\hline I & FDA approved and common in clinical practice \\
& Based on peer-reviewed literature \\
& Referred to RUC for valuation \\
II & Used to report quality performance initiatives \\
& Decreased need for records review \\
& No reimbursement \\
III & Track new/experimental procedures \\
& Literature suggests possible future use \\
& Temporary, by definition \\
\hline
\end{tabular}

Table 4: How MPPR can reduce technical and professional component reimbursement

\begin{tabular}{lcccc}
\hline & Procedure 1 & Procedure 2 & $\begin{array}{c}\text { Total } \\
\text { Payment }\end{array}$ & MPPR Applied \\
\hline TC & $\$ 500$ & $\$ 400$ & $\$ 900$ & $\$ 700(\$ 500+[\$ 0.50 \times \$ 400])$ \\
PC & $\$ 100$ & $\$ 80$ & $\$ 180$ & $\$ 160(\$ 100+[\$ 0.75 \times \$ 80])$ \\
Global & $\$ 600$ & $\$ 480$ & $\$ 1080$ & $\$ 860$ \\
\hline
\end{tabular}

examinations defined as sonography, CT, CT angiography, MR imaging, and MR angiography. In this practice, Medicare will fully reimburse for the most expensive examination when multiple imaging examinations are performed on a single patient by the same physician or group during a single health care encounter (single patient session), but it will reduce reimbursements for subsequent imaging examinations performed during that encounter.

This practice was implemented in a graded manner during several years, ${ }^{4}$ with a $25 \%$ reduction in 2006 to the TC of advanced imaging examinations of adjacent anatomy performed by the same physician, on the same patient, on the same day. In 2010, this reduction increased to $50 \%$ through the Affordable Care Act. MPPR was subsequently modified in 2011 by the CMS to include nonadjacent anatomy across different imaging modalities (regardless of the relevant code family). In other words, the imaging MPPR was modified to apply to multiple imaging services furnished within the same family of codes or across code families, currently applying to CT and CTA, MR imaging and MRA, and sonography services furnished to the same patient in the same session, regardless of the imaging technique, and not limited to contiguous body areas. Additional regulations were imposed in 2012, expanding the MPPR by cutting the PC by $25 \%$. In 2013, the MPPR was further broadened by the CMS to apply to physicians in the same group practice (same Group National Provider Identifier) caring for the same patient on the same day. It is estimated that the total losses in both TC and PC imaging fee reimbursement due to the implementation of the MPPR policy were approximately $\$ 1.2$ billion from 2006 to 2013 . $^{4}$

According to the CMS, the primary rationale for these cuts to reimbursement related to redundant work within both the TC and PC, when patients undergo multiple imaging examinations during a single day. ${ }^{4}$ The justification for reducing the TC reimbursements related to activities that are not repeated during multiple examinations on the same patient during the same encounter, such as preparing and cleaning the examination room, educating the patient, obtaining informed consent, placing an IV line, and so forth. However, applying MPPR to a radiologist's interpretation (PC) of multiple imaging examinations is particularly controversial, because the radiologist is responsible for all subsequent images when multiple examinations are performed and these follow-up examinations are often as timeintensive as the initial examination. See Table 4 for an example of how MPPR can reduce technical and professional component reimbursement.

Code Bundling. With the development of new bundled CPT codes, further reductions in radiology reimbursement have been seen. The American Medical Association Specialty Society Relative Value Scale Update Committee (RUC) has pinpointed "potentially misvalued" services, which are defined as services performed and billed together with varying degrees of overlap or redundancy in time and effort. On the basis of methods provided by the CMS and the RUC, codes have been identified that have a history of being billed together 95\%, $90 \%$, and $75 \%$ of the time (and more 
recently $50 \%$ of the time), which often result in the creation of new revalued "bundled" codes. This practice, in turn, initiates a controversial cascade of payment reductions for the TC, PC, and hospital, with often little advanced notice between notification and implementation (approximately 3 months). ${ }^{4}$

The ASNR has played a proactive role in neuroradiology-related code valuation and revaluation recommendations brought before the RUC. ${ }^{7}$ One prototypical procedure usually performed by neuroradiologists flagged by the "codes performed together screen" was myelography and the near-universal association among injection, supervisory, and interpretation codes. The ASNR, along with the American College of Radiology, revised the code set and presented both the new bundled code sets and the original free-standing codes to the RUC for valuation. Four new bundled codes were introduced in CPT 2015 for myelography with the same physician performing and supervising the procedure and interpreting the images. If separate providers perform the procedure and interpret the images, then the nonbundled codes are used.

Another example from 2010 involved carotid/cerebral angiography, for which a plethora of related procedure codes were flagged by the screen describing catheterization and injection as well as radiologic supervision and interpretation. As a result, after input from multiple stakeholders including the ASNR, a new series of codes were proposed and subsequently approved by the CPT Editorial Panel in 2012, which were then forwarded to the RUC for valuation. This revision, nonetheless, resulted in a substantial decrease in RVUs beginning in January 2013, which, in turn, caused physician reimbursement to plummet. A single-vessel selective diagnostic angiogram of an internal carotid artery that had a CMS valuation of 7.6 RVUs in 2012 would be replaced with a single bundled code for the procedure and interpretation valued at 6.5 RVUs in 2013, a reduction of 15\%. A standard 4-vessel angiogram valued at 18.22 RVUs decreased to 14.25 RVUs, a reduction of $22 \%$.

Bundling of codes has probably had the greatest impact in mammography. As a result, an entire group of breast interventions has been condensed into 14 new bundled codes, resulting in measurable reductions to the TC and PC across this group of interventions. Stereotactic breast biopsy alone has undergone reductions of up to $45 \%$ for the PC and up to $3 \%$ for the TC. ${ }^{4}$ This reduction has untoward consequences when attempting to recruit trainees into breast imaging fellowships and radiologists into breast imaging careers/positions, not to mention its impact on retaining patients within a given radiologist's health care system.

In addition to myelography and carotid/cerebral angiography noted above, areas of neuroradiology most affected by code bundling include vertebroplasty/vertebral augmentation, scoliosis plain film series, and fetal MR imaging, to name a few. As CPT code families continue to be screened by the Relativity Assessment Workgroup (discussed later in this article) or CMS, the ASNR continues to represent the interests of neuroradiology. In cooperation with the CPT Editorial Panel, the ASNR has been instrumental in crafting new coding proposals for these procedures (and others) in their ongoing advocacy for the subspecialty.

Bundled Payments for Care Improvement Initiative. The Bundled Payments for Care Improvement Initiative offers providers a single combined reimbursement for a single illness or "episode of care" in an Accountable Care Organization, which seeks to incentivize higher value care and more effective coordination of providers across multiple settings via alternative payment models. ${ }^{9}$ By offering a bundled payment, the assumption is that providers will develop their own internal controls and become jointly accountable for each episode of care; these results will cause providers to assume greater risk as costs burgeon compared with the aging fee-for-service model. This form of payment bundling is separate and distinct from the CPT code bundling discussed above, which refers to the redefining of multiple CPT codes for services often reported together in a single CPT code for a given combination of services. Both CPT code bundling and payment (or procedure) bundling have a similar effect, to reduce overall payment for a given combination of services.

The Bundled Payments for Care Improvement initiative seeks to align incentives for providers across a broad spectrum of medical and paramedical services provided by physicians, hospitals, nonphysician providers, and other health care facilities under 4 basic models: 1) acute care inpatient hospitalization with a standard discount related to Medicare Part A inpatient payments, 2 and 3 ) retrospective bundling of payments in which expenditures are reconciled against a target price for each episode of care, and 4) a prospective bundled payment scheme in which a lump sum payment is made to providers for the entire episode of care. ${ }^{9}$ Each of these models has a different impact on providers, from maintaining status quo payment under the current fee-for-service system for model 1 to varying degrees of payment bundling, with model 4 ("prospective bundling") being the most ambitious with its single prospectively determined reimbursement made to the hospital to cover all services that occur during a single episode of care.

\section{Level 2: Cost-Savings Initiatives}

Time-Driven Activity-Based Costing. Time-Driven Activity-Based Costing (TDABC) is a strategic accounting and profitability tool used by many businesses, which can be applied by health care providers to monitor the cost of a patient's treatment pathway. ${ }^{10}$ Combining this tool with the patient's outcome data provides a means to more objectively measure the value of care. It reveals to clinicians the underlying factors that drive costs and functions across a broad spectrum of medical settings and organizations. Clinicians are then able to identify variation in costs among similar providers across different pathways for the same medical services. For example, when one compares the average cost of primary total knee arthroplasty for an organization, the 90th percentile of total cost is nearly twice the average for an organization at the 10th percentile of total cost using the TDABC approach. ${ }^{10}$ TDABC only applies to a practice or departmental effort to control its own costs and is independent of coding, billing, and reimbursement.

Current costing mechanisms lack standardization, given a patient's treatment plan, which often involves several departments, none of which use a standard treatment pathway for patients with similar illnesses. They often focus on only measuring and controlling costs for a small subset, such as a department or for individual procedures; this focus is not effective for reducing long-term 
costs, given the pedantic micromanaging of line-item expenses with implementation of arbitrary limits on department spending, which can greatly reduce the value and quality of services provided. ${ }^{10}$ These accounting problems are further exacerbated by the reimbursement systems and accounting practices currently in place at many health care organizations.

As mentioned above, many providers use RVUs to allocate clinical departmental costs to various procedures and treatments; this use tends to introduce substantial distortions into cost measurement. This allocation is problematic when attempting cost stratification, given the highly subjective judgments and aggregate data on which RVUs are based and the difficulty of validating them in actual clinical practice. Only reimbursed procedures and processes have RVUs assigned to them, not unreimbursed ones; therefore, they do not reflect the use of different staff and personnel with widely different compensation, thereby confusing procedure complexity with the resource time required to perform the procedure.

There are several foundational principles for measuring costs in health care, and TDABC provides a means for enabling each of them. ${ }^{10}$ First, the cost of using a resource, whether personnel or equipment, depends only on the time the resource is in use and the price rate for that resource, even with varying reimbursement for different services. The cost of a clinician is the same if the time required for the procedure is the same, whether performing a high-RVU procedure or a low-RVU procedure. Second, the unit of analysis for measuring costs and outcomes should be the patient's medical condition. Third, costs should be measured over the entire treatment cycle for a given medical condition, including diagnosis, tests, education/counseling, interventions, and managing ongoing care. Fourth, if a resource is not used, it should be considered unused and not assigned to the services provided.

TDABC, a well-established method of cost measurement across multiple industries, ${ }^{10}$ calculates the cost of resources used to perform a procedure or treat a patient across the care cycle by estimating the cost (rate) of each used resource and the time it is consumed over the full cycle of the patient's care for a particular medical condition. This is facilitated by creating a process map of the care cycle or procedure and identifying all clinical and administrative resources used to treat the specific medical condition. Various methods can be used to elicit information for the aforementioned process maps within an organization, including interviewing involved personnel about the care processes, direct observation via shadowing, data capture from the electronic medical record, and review of administrative data. When the project team can accurately measure the quantities of personnel, equipment time, and all material consumables used during the complete treatment cycle, the process map is considered complete. Next, the project team uses the payroll data of the institution to calculate the cost rate per minute of time of each resource, whether personnel, equipment, or space, and then multiplies the cost rate of each resource by the amount of time the patient spent in each resource, followed by summing of all resource cost rates to compute the total cost of the patient care cycle.

The results of a comprehensive TDABC analysis will provide hospitals and departments with valuable opportunities to intervene at multiple levels in the process; with analytic comparisons both before and after interventions, organizations will be provided the necessary information to identify and implement real and sustained reductions in costs.

Avoid Penalties: Preemptive Participation in Medicare Access and Children's Health Insurance Program Reauthorization Act. The US Department of Health and Human Services has put forth an ambitious plan for health care in the United States, focusing on "better care, smarter spending, and healthier people," seeking to tie $90 \%$ of the Medicare payment to the quality of care by 2018 , which stands in contradistinction to the current fee-for-service system. ${ }^{11}$ These principles underpin the bipartisan Medicare Access and Children's Health Insurance Program Reauthorization Act (MACRA) of 2015. MACRA seeks to achieve these goals by incentivizing quality over quantity and rewarding more efficient clinical decision-making, seeking to actuate patient-centered health care delivery that is meaningful, flexible, cost-effective, and practically feasible to improve health outcomes and the overall care experience. MACRA will be discussed in greater detail later in this article.

Another way to take part in cost saving in a practice or department is to preemptively begin laying the groundwork necessary to participate in the Merit-Based Incentive Payment System and various Advanced Alternative Payment Models, which are described in MACRA. A more complete discussion of these models will appear later in this article, but being ready to participate in these models now could result in measurable cost savings for the clinician and the practice/department. In 2019, MACRA will begin to impact physicians' Medicare Part B reimbursements. For example, under the Merit-Based Incentive Payment System clinicians will experience either negative, neutral, or positive adjustments to their traditional fee-for-service payments based on a range of performance measures. ${ }^{11}$

Practical Departmental Measures. Devising an evolving plan to address ongoing departmental cost-saving initiatives is not too difficult if one is willing to be proactive and use a little imagination. One way to take part is to actively identify departmental waste and approve a standing policy to correct it. For example, when one performs a procedure such as a lumbar puncture, it is more cost-efficient to use the vial of lidocaine already provided in the lumbar puncture kit as opposed to drawing from a separate larger external vial that may make the process slightly easier. A 20 - $\mathrm{mL}$ bottle of lidocaine $\mathrm{HCl} 1 \%(10 \mathrm{mg} / \mathrm{mL})$ can range anywhere from approximately $\$ 2.00$ to $\$ 3.00$ per bottle, depending on the manufacturer/distributor. Another cost-saving solution might be to obtain bicarbonate separately (if commonly used in the department/practice) and manually mix it with the lidocaine rather than ordering a lidocaine-bicarbonate preparation from the hospital pharmacist. This general idea can be applied across the board for all departmental procedures - that is, using items within the provided kits and exercising greater discretion as to when to use additional items that basically have the same functionality.

\section{Level 3: Coding and Billing for Subspecialty-Specific Examinations and Reports}

Report Elements That Support Examination Coding/Billing. Several key elements in the structured radiology report are necessary not only to meet published American College of Radiology Prac- 
Table 5: Required content and noncontent attributes

\section{Content Items}

History (with relevant information)

Technique (list procedures/materials)

Exam quality (degradation factors)

Description

Comparison

Diagnosis

Differential (when appropriate)

Address clinical question

Recommendations (when appropriate)

Conclusions

Table 6: Examples of the differences between ICD-9 and ICD-10 codes

\begin{tabular}{ccc}
\hline Example & ICD-9 & ICD-10 \\
\hline Neuroradiology & $\begin{array}{c}\text { 852.21 Subdural hemorrhage } \\
\text { without coma }\end{array}$ & $\begin{array}{c}\text { S06.5X0A Traumatic subdural } \\
\text { hemorrhage without loss of } \\
\text { consciousness, initial encounter }\end{array}$ \\
& c13.45 Torus fracture of & S52.521A Torus fracture of lower end \\
radius (alone) & of right radius, initial encounter \\
& for closed fracture
\end{tabular}

Examples of Neuroradiology-Specific CPT Codes with RVU Values. The following are examples of neuroradiologyspecific CPT codes with their respective CMS RVU values ${ }^{6}$ to further highlight the individual components of each code and the relative weights of the technical and professional components of the global fee. Remember ICD codes relate to the classification of disease (patient clinical presentation), whereas CPT codes relate to the coding of diagnostic procedures, such as MR imaging (below).

CPT Code 70551: "MR Imaging Brain without Contrast." In the Washington, DC area, CPT code 70551 is assigned 6.52 RVUs for the global payment, 4.41 RVUs for the technical component, and 2.11 RVUs for the pro-

tice Parameters, recommendations and standards that were evaluated using the Appraisal of Guidelines for Research and Evaluation tool, but also to support successful examination coding and billing. ${ }^{12-14}$ The Appraisal of Guidelines for Research and Evaluation tool assesses the quality of guidelines (including the methodologic rigor and transparency with which a guideline is developed), provides a methodologic strategy for the development of guidelines, and outlines what and how information ought to be reported in guidelines. ${ }^{15}$ There are both content and noncontent attributes that should be appropriately reflected in a typical radiology report. Required content and noncontent attributes are included in Table $5 .^{12}$

Additionally, some items need to be included in the written radiology report to make it International Statistical Classification of Diseases and Related Health Problems-10 compliant (ICD-10; https://www.cms.gov/Medicare/Coding/ICD10/). ICD-10 is the 10th revision of the International Classification of Diseases (ICD), a medical classification list by the World Health Organization containing codes for various diseases, as well as signs and symptoms. ${ }^{16}$ With ICD-10 codes, the amount of information required from the referring physician has increased dramatically compared with ICD-9 (the older ninth revision). Imaging facilities need to acquire a more complete history for a patient when scheduling an examination for precertification, dictation, and subsequent billing of a procedure. Important elements include a more specific clinical diagnosis and disease acuity, detailed anatomic site, secondary or tertiary diagnoses, laterality (eg, rightarm numbness), specifying initial-versus-subsequent encounters, and detailed procedure descriptions (when applicable). It is important to include as much clinical information as possible to help ensure compliance and avoid the pitfalls of being too brief. For example, it is insufficient to state "concern for infarct" in the indications section of the report. A more appropriate phrase might be "worsening right arm and hand weakness over the last hour, concern for acute infarct."

See Table 6 for examples that highlight the differences between ICD-9 and ICD-10 codes. ${ }^{17,18}$ fessional component (1.48 RVUs for physician work) from the Medicare code key.

CPT Code 72148: "MR Imaging Lumbar Spine without Contrast." In the Washington, DC area, CPT code 72148 is assigned 6.32 RVUs for the global payment, 4.19 RVUs for the technical component, and 2.13 RVUs for the professional component (1.48 RVUs for physician work) from the Medicare code key.

\section{CONCLUSIONS}

The tapestry of health care economics as it applies practically to radiologists is complex, with relatively fragmented dissemination in the current medical literature. Therefore, we have presented a tailored discussion in the form of a study guide for fellows to learn from and gain competence in the ACGME neuroradiology milestones on health care economics. While this article is targeted to neuroradiology fellows, it can be useful for others in the radiologic sciences and medicine as a whole. While not meant to be exhaustive, our aim is that this review article might serve as a foundation on which radiology residents, imaging subspecialty fellows, practicing radiologists, and other medical and allied health care professionals can build upon, facilitating their implementation in real-world radiology/clinical practice.

Because health care economics is a constantly evolving entity, the following Web sites are provided as resources to follow some of the latest changes in the health care economics landscape:

CPT information: https://www.ama-assn.org/practicemanagement/cpt-current-procedural-terminology; and https:// www.ama-assn.org/practice-management/explore-recent-cptcode-changes-actions

MACRA Merit-Based Incentive Payment System information: https://www.acr.org/Quality-Safety/Resources/MACRA-Resources

Medicare information: https://www.cms.gov/Medicare/ Medicare.html

RUC information: https://www.ama-assn.org/about-us/ruc. 


\section{REFERENCES}

1. The Accreditation Council for Graduate Medical Education and The American Board of Radiology. The Neuroradiology Milestone Project. Updated 2015. https://www.acgme.org/Portals/0/PDFs/Milestones/ NeuroradiologyMilestones.pdf. Accessed April 30, 2017

2. Donovan WD. The resource-based relative value scale and neuroradiology: ASNR's history at the RUC. Neuroimaging Clin N Am 2012;22:421-36 CrossRef Medline

3. Rimler SB, Gale BD, Reede DL. Diagnosis-related groups and hospital inpatient federal reimbursement. Radiographics 2015;35: 1825-34 CrossRef Medline

4. Baadh A, Peterkin Y, Wegener M, et al. The relative value unit: history, current use, and controversies. Curr Probl Diagn Radiol 2016; 45:128-32 CrossRef Medline

5. Centers for Medicare and Medicaid Services. PFS relative value files. Updated 2016. https://www.cms.gov/medicare/medicare-fee-forservice-payment/physicianfeesched/pfs-relative-value-files.html. Accessed May 16, 2017

6. Centers for Medicare and Medicaid Services. Physician fee schedule search. Updated 2016. https://www.cms.gov/apps/physician-feeschedule/search/search-criteria.aspx. Accessed May 16, 2017

7. Leslie Mazwi TM, Bello JA, Tu R, et al. Current procedural terminology: history, structure, and relationship to valuation for the neuroradiologist. AJNR Am J Neuroradiol 2016;37:1972-76 CrossRef Medline

8. Petrey WB, Allen B Jr, Thorwarth WT Jr, et al. Radiology coding, reimbursement, and economics: a practical playbook for housestaff. J Am Coll Radiol 2009;6:643-48 CrossRef Medline

9. Hirsch JA, Leslie-Mazwi TM, Barr RM, et al. The bundled payments for care improvement initiative. J Neurointerv Surg 2016;8:547-48 CrossRef Medline
10. Oklu R, Haas D, Kaplan RS, et al. Time-driven activity-based costing in IR. J Vasc Interv Radiol 2015;26:1827-31 CrossRef Medline

11. Rosenkrantz AB, Nicola GN, Allen B Jr, et al. MACRA, alternative payment models, and the physician-focused payment model: implications for radiology. J Am Coll Radiol 2017;14:744-51 CrossRef Medline

12. Pool F, Goergen S. Quality of the written radiology report: a review of the literature. J Am Coll Radiol 2010;7:634-43 CrossRef Medline

13. Fleming M, MacFarlane D, Torres WE, et al. Magnitude of impact, overall and on subspecialties, of transitioning in radiology from ICD-9 to ICD-10 codes. J Am Coll Radiol 2015;12:1155-61 CrossRef Medline

14. American Medical Association. Fact sheet: The differences between ICD-9 and ICD-10. https://www.unitypoint.org/waterloo/filesimages/for\%20providers/icd9-icd10-differences.pdf. Accessed May 16, 2017.

15. The AGREE Next Steps Consortium. Appraisal of guidelines for research and evaluation II instrument. Updated 2013. http://www. agreetrust.org/wp-content/uploads/2013/10/AGREE-II-Users-Manualand-23-item-Instrument_2009_UPDATE_2013.pdf. Accessed June 14, 2017

16. World Health Organization. Classification of diseases (ICD). Updated 2016. http://www.who.int/classifications/icd/en/. Accessed June 14, 2017

17. Centers for Medicare and Medicaid Services. ICD-9-CM diagnosis and procedure codes: Abbreviated and full code titles. Updated 2014. https:/www.cms.gov/medicare/coding/ICD9providerdiagnosticcodes/ codes.html. Accessed May 16, 2017

18. Centers for Medicare and Medicaid Services. 2017 ICD-10-CM and GEMs. Updated 2016. https://www.cms.gov/Medicare/Coding/ICD10/ 2017-ICD-10-CM-and-GEMs.html. Accessed May 16, 2017 